\title{
Sur la rencontre de deux visages du socialisme européen
}

Les influences du socialisme belge sur le socialisme français, la

coopération (1885-1914)

\section{The Meeting of Two Facets of European Socialism}

The Impact of Belgian Socialism on French Socialism Seen

Through the Cooperative Movement (1885-1914)

\section{Daniel Ourman}

Numéro 280, avril 2001

URI : https://id.erudit.org/iderudit/1023671ar

DOI : https://doi.org/10.7202/1023671ar

Aller au sommaire du numéro

Éditeur(s)

Institut de l'économie sociale (IES)

ISSN

1626-1682 (imprimé)

2261-2599 (numérique)

Découvrir la revue

Citer cet article

Ourman, D. (2001). Sur la rencontre de deux visages du socialisme européen : les influences du socialisme belge sur le socialisme français, la coopération (1885-1914). Revue internationale de l'économie sociale, (280), 80-91.

https://doi.org/10.7202/1023671ar

\section{Résumé de l'article}

Si l'influence française sur le socialisme belge s'est exercée durant une bonne partie du XIX ${ }^{\mathrm{e}}$ siècle, l'auteur s'interroge sur l'existence d'une influence du socialisme belge sur celui de France, en considérant la période qui sépare la création du Parti ouvrier belge (POB) de la Première Guerre mondiale. Le champ précis de la coopération, dont le poids au sein du POB constituait l'un des traits caractéristiques du mouvement socialiste belge, offre un éclairage intéressant à cette question. L'émigration temporaire ou définitive de travailleurs belges en France, le voyage en Belgique des socialistes français, la collaboration commune à des revues représentent trois modalités de contacts directs entre socialistes des deux pays. Le modèle de la coopération belge attire alors l'attention de socialistes français qui, bien souvent, s'en inspirent. Ainsi, de 1885 à 1914, leur opinion sur la coopération évolue sensiblement sous l'influence du modèle belge et de ses deux plus beaux fleurons : le Vooruit de Gand et la maison du peuple de Bruxelles. 


\title{
Sur la rencontre de deux visages du socialisme européen LES INFLUENCES DU SOCIALISME BELGE SUR LE SOCIALISME FRANÇAIS: LA COOPÉRATION (1885-1914)
}

\author{
par Daniel Ourman")
}

${ }^{*}$ ) Daniel Ourman est professeur certifié d'histoire et géographie.
Si l'influence française sur le socialisme belge s'est exercée durant une bonne partie du XIX siècle, l'auteur sinterroge sur l'existence d'une influence du socialisme belge sur celui de France, en considérant la période qui sépare la création du Parti ouvrier belge (POB) de la Première Guerre mondiale. Le champ précis de la coopération, dont le poids au sein du POB constituait l'un des traits caractéristiques du mouvement socialiste belge, offre un éclairage intéressant à cette question. L'émigration temporaire ou définitive de travailleurs belges en France, le voyage en Belgique des socialistes français, la collaboration commune à des revues représentent trois modalités de contacts directs entre socialistes des deux pays. Le modèle de la coopération belge attire alors l'attention de socialistes français qui, bien souvent, s'en inspirent. Ainsi, de 1885 à 1914, leur opinion sur la coopération évolue sensiblement sous l'influence du modèle belge et de ses deux plus beaux fleurons: le Vooruit de Gand et la maison du peuple de Bruxelles.
(1) Cet article s'appuie sur un mémoire de DEA réalisé durant I'année universitaire 1993-1994, sous la direction de M. le professeur Jacques Julliard, dans le cadre de la formation doctorale " Histoire et civilisations " de l'EHESS.

(2) Bruxelles a été, au XIX siècle, I'un de ces lieux de refuge où vivaient d'innombrables émigrés et qui ont favorisé les interpénétrations entre les diverses familles socialistes européennes. Les Français, qui ont été les plus nombreux à s'y réfugier, ont participé aux débats idéologiques et ont contribué à faire de la ville belge un véritable bouillon de culture politique, la communauté de langue - Bruxelles, ville bilingue, ne cessa de se franciser - facilitant les échanges, notamment entre socialistes des deux pays. 'histoire internationale du mouvement ouvrier est en soi une dimension essentielle, complexe et riche, mais aussi une façon d'aborder l'évolution du socialisme. Cette attention que l'historien du mouvement ouvrier se doit de porter à la dimension internationale nous a guidé dans l'étude des relations qu'ont entretenues socialistes français et belges entre 1885 et $1914^{(1)}$. En effet, au-delà du rôle qu'a joué Bruxelles - au moins à partir de 1848 - dans l'osmose des hommes et des idéologies ${ }^{(2)}$, il nous a paru intéressant de nous interroger plus généralement sur l'interaction entre socialismes belge et français durant la période qui s'étend de 1885 à 1914 . Or, lorsqu'il est question des relations privilégiées qu'ont entretenues les socialistes des deux pays, l'idée qui vient d'abord à l'esprit est celle d'une influence des Français sur leurs camarades d'outre-Quiévrain. Il semble, en effet, que le mouvement socialiste belge ait été fortement marqué, jusqu’à la disparition de la I ${ }^{\text {re }}$ Internationale (1876), par des idéologies d'origine étrangère et tout particulièrement française - qu'il s'agisse des doctrines de Saint-Simon, Fourier, Louis Blanc ou surtout Proudhon. Si l'influence française sur le mouvement socialiste belge s'est exercée durant une bonne partie du XIX ${ }^{e}$ siècle, qu'en a-t-il été par la suite? 
Doit-on considérer que les relations entre les socialistes des deux pays ont toujours pris cet aspect? Ne convient-il pas, au contraire, de s'attacher au caractère réciproque que recèle toute interaction? Cette réciprocité est déjà présente dans les facteurs géographique et linguistique qui contribuent à expliquer l'influence française: la proximité comme l'identité de langue valent pour l'un et l'autre pays. Nous pouvons supposer, en outre, que les échanges entre les socialistes français réfugiés et leurs hôtes belges n'ont pas revêtu un caractère purement univoque. Bref, ne doit-on pas réfléchir à une influence du socialisme belge sur celui de France? C'est le choix que nous avons fait. Considérant la période qui sépare la création du Parti ouvrier belge (POB) de la Première Guerre mondiale, nous nous sommes interrogés sur l'existence de cette influence. Pour cela, nous avons utilisé un champ bien précis: la coopération, dont le poids au sein du POB constituait l'un des traits caractéristiques du mouvement socialiste belge. Si une influence s'est effectivement développée, dans quels termes s'est-elle posée? Quels canaux, quels vecteurs a-t-elle empruntés pour s'exprimer? Quels effets a-t-elle eus? Comprendre ce système de relations, d'interactions, c'est aussi se demander si les influences belges ont eu un ou des terrains de prédilection; c'est aussi s'interroger sur les liens spécifiques entre socialisme du nord de la France et socialisme belge: dans quelle mesure des environnements économiques et socioculturels proches, les phénomènes de migration entre les deux pays ont-ils pesé?

\section{Du traitement de la question des influences}

(3) J. Julliard, "Sur un fascisme imaginaire: à propos d'un livre de Zeev Sternhell ", Annales ESC, $n^{\circ} 4$, juillet-août 1984, p. 852.

(4) Ainsi, dans sa thèse, Claude Willard s'interrogeait sur le rôle qu'ont joué les guesdistes dans l'introduction du marxisme en France, dans la connaissance et la diffusion des œuvres du penseur allemand (C. Willard, Le mouvement socialiste en France [1893-1905]: les guesdistes, Editions sociales, Paris, 1965). Traitant à son tour de cette question, Christophe Prochasson s'arrêtait au cas de Charles Andler (C. Prochasson, "Sur la réception du marxisme en France ", loc. cit., p. 108). Au-delà, cette étude lui permettait de s'interroger sur les modalités de diffusion d'une doctrine.
Si elle nous semble particulièrement importante pour une histoire qui prend pleinement en considération les phénomènes d'échanges, d'interactions, la question des influences n'a pas toujours été traitée avec bonheur et la rigueur nécessaire par les historiens qui l'abordèrent. Quand toute la démarche de l'historiographie récente tend à privilégier l'analyse des comportements concrets, quand elle tend à contrebalancer l'étude du discours par celle de la pratique, certains continuent à "prendre ce dernier pour argent comptant ${ }^{(3)}$ ". Or, les méthodes herméneutiques de l'histoire intellectuelle doivent amener à une réinsertion des idées dans leur environnement social et culturel; elles doivent conduire l'historien à remettre les idées en situation dans un contexte historique. Il s'agit, pour cette histoire, de s'ouvrir de plus en plus aux phénomènes de diffusion, de réception et de mise en place des idées, à l'image du travail qui a été effectué sur l'histoire de la réception du marxisme en France ${ }^{(4)}$. Il est à souligner que l'histoire intellectuelle du socialisme a pu bénéficier des travaux de chercheurs tels que Madeleine Rebérioux et Daniel Lindenberg, qui ont tous deux contribué à son décloisonnement. La réflexion sur les concepts de "diffusion ", de " réception ", d' " influence " a été, quant à elle, grandement nourrie par les travaux d'historiens qui se retrouvent, par ailleurs, dans la démarche qu'ont empruntée les Cahiers Georges Sorel puis la revue Mil neuf cent. 
(5) Revue de synthèse, "Réception et contresens ", 1, janvier-mars 1989, cité par C. Prochasson, " Histoire intellectuelle, histoire des intellectuels: le socialisme français au début du XX" siècle ", Revue d'histoire moderne et contemporaine, 39-3, juillet-septembre 1992, p. 437.

(6) Chaque année, 40000 à 50000 Belges arrivaient dans le centre du Bassin parisien, fournissant les deux tiers de la main-d'œuvre au moment des moissons.

(7) F. Lentacker, "Les Frontaliers belges travaillant en France: caractères et fluctuations d'un courant de main-d'œuvre ", Revue du Nord janvier 1950.
S'il ne saurait y avoir d'histoire intellectuelle sans " histoire des réceptions, sans histoire des "orthodoxies" et des "contresens", mis à égal niveau "(5) ", la mise en évidence d'influences constitue, quant à elle, le problème clé de l'histoire intellectuelle. Nous nous sommes efforcé d'évaluer dans quelle mesure des influences ont joué entre les mouvements socialistes belge et français. Plus précisément, nous avons tenté d'apporter des éléments de réponse à la question de l'influence des socialistes belges sur ceux de France dans le domaine de la coopération. Nous avons cherché à cerner ces influences, nous nous sommes interrogé sur leurs modalités, sur les canaux d'irrigation qu'elles ont empruntés.

\section{Les vecteurs d'influences}

Emigration temporaire ou définitive et donc présence - importante - de travailleurs belges en France, voyage en Belgique des socialistes français, collaboration commune à des revues constituant de potentiels lieux d'échanges: ces trois modalités de contacts directs entre socialistes des deux pays nous ont paru particulièrement éclairantes et nous ont amené à nous interroger sur leur qualité de vecteurs d'influences.

\section{Les travailleurs belges en France}

En 1885, les Belges représentaient à eux seuls plus de $40 \%$ de l'ensemble des étrangers vivant en France. Et au-delà, ils ont constitué, parmi la population immigrée, le groupe le plus important au XIXe siècle. Cet afflux est le produit d'une situation économique et sociale difficile, parfois tragique, en Belgique tout au long du XIX siècle. Nombreux furent les départs, saisonniers ou définitifs, vers cette France qui, dans les dernières décennies du XIX siècle, se présentait comme un débouché normal. Des milliers d'agriculteurs flamands - ouvriers saisonniers (les "franschmans") - se rendaient chaque année dans de grandes entreprises agricoles du nord de la France et d'Ile-de-France ${ }^{()}$. Par ailleurs, d'importants contingents ouvriers, partant des communes belges proches de la frontière, allaient chercher du travail dans les usines françaises. Ils arrivaient le lundi matin et rentraient chez eux le samedi soir avec leur salaire. De temporaires, les migrations des saisonniers belges se transformaient fréquemment en une immigration définitive. Bon nombre de Belges finirent par se fixer en France. Ainsi que le font le plus souvent les étrangers, les Belges ont eu tendance à se concentrer dans les départements frontières, proches de leur pays, si bien que sur un total de 465000 Belges vivant en France en 1891, 340000 se trouvaient dans les départements du Nord, du Pas-de-Calais et des Ardennes.

A travers ces flux migratoires apparaitt l'une des dimensions de la réalité frontalière; réalité particulièrement illustrée par la frontière franco-belge. Firmin Lentacker ${ }^{(7)}$ a souligné le caractère artificiel et perméable de la frontière d'Etat séparant depuis 1814 le nord de la France de la Belgique. L'établissement de la frontière n’a jamais rompu les relations entre Français et 
(8) Ils échappaient ainsi à la surveillance de la police française, qui ne pouvait pénétrer en territoire étranger.

(9) Participèrent notamment à ces réunions Henri Carette et Albert Lepers, qui devaient s'emparer plus tard de la mairie de Roubaix.

(10) M. Hastings, Halluin la Rouge, 1919-1939, Presses universitaires de Lille, Lille, 1991, p. 133.

(11) Menin est une ville de Belgique qui, située sur la Lys, se trouve à la frontière française.
Belges. Simplement, les hommes durent en tenir compte pour mieux s'y adapter; les rapports des populations s'organisèrent en fonction de cette ligne de démarcation conventionnelle.

Ainsi le XIX siècle vit-il se développer et s'intensifier une vie frontalière née de la fraude. Et, dans la seconde moitié du siècle, l'organisation traditionnelle de la fraude épicière fut reprise à des fins politiques dans le cadre de la propagande socialiste qui se développa depuis la Belgique, s'intensifiant progressivement. Dans un premier temps, elle fut principalement, voire exclusivement, menée par des proscrits français qui profitaient de la protection que leur offrait une législation belge particulièrement libérale (libertés de presse et de réunion). Pour ce faire, un vaste système contrebandier se mit en place. Puis les organisations belges commencèrent à encadrer certains de ces militants français, qui paraissaient isolés avant 1864, et à faciliter considérablement leur action. Aux Français se joignirent les propagandistes belges dont le mouvement ouvrier commençait à se développer. Certains, tels Edouard Anseele et Edmond Van Beveren, venaient de Gand tous les quinze jours, voire tous les dimanches, pour tenir des réunions secrètes dans un estaminet des faubourgs de Tourcoing, mais en territoire belge ${ }^{(8)}$. Ils faisaient partie de ces prédicateurs flamands qui parcouraient inlassablement les cités frontalières situées en Belgique. D'autres réunions de ce genre se tenaient près d'Armentières, d'Halluin ou encore de Menin. Elles étaient l'occasion d'inciter les ouvriers à organiser des grèves et des sociétés de résistance. Ainsi, ce fut en partie sous l'impulsion de militants socialistes belges que se formèrent peu à peu certains dirigeants socialistes du Nord ${ }^{\left({ }^{(9)}\right.}$. Les réunions et la distribution de publications révolutionnaires ne constituaient d'ailleurs pas les seules manières de faire de la propagande. Il arrivait aussi que des ouvriers roubaisiens soient conviés à assister à des meetings outre-Quiévrain. Ainsi la situation frontalière du département du Nord permit-elle aux socialistes belges de développer vers la France la diffusion de leur propagande. Quant aux cités frontalières, leur situation géographique ainsi que les conditions historiques de leur peuplement les ont orientées vers la Belgique.

Dans l'ouvrage qu'il a consacré à Halluin la Rouge, Michel Hastings souligne le rôle inducteur des travailleurs belges dans la germination des idées socialistes et l'organisation du mouvement ouvrier local: «L'éclosion des idées socialistes [s'est faite] à travers la double filiation du mouvement ouvrier belge et du guesdisme roubaisien qui [définirent] les structures et les méthodes de fonctionnement des premières organisations ${ }^{(10)}$. "Dans cette cité de 15000 habitants située à la frontière franco-belge et entièrement organisée autour d'une mono-industrie textile, le rôle inducteur des Flamands lui semble irréfutable. Il se révéla notamment lors du mouvement de grève de 1895-1896. Ces Belges étaient tous des socialistes appartenant à la section meninoise ${ }^{(11)}$ du Vooruit de Gand. Ainsi retrouve-t-on cette influence du mouvement ouvrier gantois sur les travailleurs émigrés flamands et, par l'intermédiaire de ces derniers, sur le mouvement ouvrier du nord de la France. 
(12) Cf. J. Gaumont, Histoire générale de la coopération en France, Paris, 1923, et P. Brisson, E. Poisson, " La coopération " dans l'Encyclopédie socialiste, syndicale et coopérative de I'Internationale ouvrière, publiée sous la direction de Compère-Morel, Aristide Quillet, Paris, 1913. (13) A côté de La Paix de Roubaix et de L'Union de Lille, citons L'Avenir des ouvriers d'Armentières, L'Union d'Houplines, La Fraternelle d'Halluin, sans parler des coopératives de Tourcoing, Wattrelos ou Saint-Amand.

(14) Traduction littérale: "En avant ".

(15) J. Michel, Le mouvement ouvrier chez les mineurs d'Europe occidentale (Grande-Bretagne, Belgique, France, Allemagne): étude comparative des années 1880 à 1914, thèse préparée sous la direction d'Yves Lequin, université de Lyon-II, 1987.
Sans généraliser le cas halluinois, il nous semble que le socialisme belge a eu, par l'intermédiaire des travailleurs belges des usines textiles du Nord, une influence certaine sur le mouvement socialiste du nord de la France. Il apparaît que les travailleurs belges jouèrent un rôle inducteur, ayant apporté avec eux des idées - socialistes -, des pratiques, une expérience. Privés de citoyenneté électorale, ils se tournèrent, dans les années 1880, vers l'action propagandiste, formant - pour une partie d'entre eux - les premiers militants de ce guesdisme dont le Nord fut une terre d'élection. Le Nord était, par ailleurs, la principale région coopérative du pays avant la Première Guerre mondiale. Le quart des coopératives françaises, de leurs adhérents et de leur chiffre d'affaires s'y concentrait en 1914, loin devant la Seine $[8 \%]^{(12)}$. Ce furent presque toutes les cités industrielles, presque toutes les villes textiles du département qui constituèrent leurs sociétés coopératives, centres de l'activité politique, syndicale, mutualiste de la classe ouvrière locale ${ }^{(13)}$.

Or, là encore se signalent le rôle inducteur des travailleurs belges et l'influence du socialisme gantois. En effet, à l'image de La Paix de Roubaix, créée en 1885, nombre des coopératives du Nord ont été fondées par des ouvriers belges immigrés, sur le modèle du Vooruit ${ }^{(14)}$ de Gand et des sociétés belges affiliées au Parti ouvrier. Identifié comme la plus prestigieuse des coopératives socialistes belges, véritable base de toute l'organisation ouvrière gantoise, le Vooruit avait été créé en 1880 par des socialistes parmi lesquels figuraient Anseele et Van Beveren. Le Vooruit constituait un modèle en Belgique, mais aussi en Allemagne (dans la Ruhr) et dans le reste de l'Europe. C'est ce modèle qu' importèrent les nombreux ouvriers flamands qui avaient émigré dans le bassin textile de Lille-Roubaix-Tourcoing. Dans ces villes textiles où souvent la moitié de la population était flamande, les Gantois ont implanté leur organisation. Quant aux hommes du Vooruit restés en Belgique, ils gardaient le contact avec ces ouvriers belges émigrés. Joël Michel ${ }^{(15)}$ a mis en valeur la démarcation entre le bassin textile du Nord et le bassin houiller du Pas-de-Calais, tout proche pourtant. Tandis que les ouvriers du textile développaient le mouvement coopératif, les mineurs étaient plutôt attachés au syndicat et largement réfractaires à la coopération. Si bien que, dans le Pas-de-Calais, le mouvement coopératif faisait pâle figure, les sociétés n'ayant qu'un rôle effacé. Pour expliquer ce fait, Joël Michel mentionne notamment le rôle - ou plutôt son absence - joué par l'immigration belge. En effet, dans le bassin houiller du Pas-de-Calais, la demande de main-d'œuvre s'est satisfaite d'un recrutement régional limité aux mineurs du Nord et aux campagnes environnantes densément peuplées. Aussi la main-d'œuvre belge représentait-elle un pourcentage insignifiant. De plus, elle venait du bassin minier du Hainaut, qui à cette époque n'avait pas de modèle d'organisation à proposer en matière coopérative, mais constituait lui-même une terre de mission pour le Vooruit et les socialistes bruxellois. Ainsi, humainement différent malgré sa proximité géographique, le bassin houiller du Pas-de-Calais est resté, contrairement aux cités textiles du Nord, hors de la zone d'influence gantoise. 
(16) La maison du peuple occupée jusqu'alors avait été inaugurée le 25 décembre 1886 et se trouvait dans I'ancienne synagogue de la rue de Bavière. Le nouvel édifice, bâti entièrement en style Art nouveau, était l'œuvre de l'architecte Victor Horta, qui figura parmi les pionniers de ce style 1900 et contribua largement à faire de Bruxelles la " capitale de l'Art nouveau ". La maison du peuple est restée le plus célèbre de ses ouvrages. Elle a pourtant été détruite en 1964 (17) Rapport des délégués de la Fédération des chambres syndicales ouvrières de la Seine-Inférieure à l'inauguration de la maison du peuple de Bruxelles, $20 \mathrm{p}$.

\section{Le voyage en Belgique}

Dans le sens France-Belgique, il existe un mode de relations qui, sans mobiliser - et de loin - des foules aussi importantes que l'émigration belge dans notre pays, a néanmoins représenté un type de contact direct intéressant entre les deux mouvements socialistes. Il s'agit du voyage. Le contact est ici différent de celui qui s'opère sur le territoire français. Il n'est pas question, en l'occurrence, de masses prolétarisées entrant en contact avec l'ensemble de la société française, à laquelle elles ont tendu peu à peu à s'intégrer, mais bien plutôt de militants se rendant dans un pays étranger le temps - bien plus court - d'un voyage d'études, d'une visite, afin de se rendre compte sur place de l'œuvre accomplie, de constater de visu les résultats obtenus par les socialistes belges. Ainsi des coopérateurs socialistes français sont-ils allés en Belgique pour visiter des coopératives, des maisons du peuple ou participer à l'inauguration d'un nouvel établissement et pour s'informer de l'organisation du mouvement coopératif d'outre-Quiévrain. Ces voyages trouvaient leur prolongement dans les récits, les comptes rendus que, de retour dans leur pays, les socialistes français donnaient à leurs camarades. Ainsi, ils retranscrivaient leurs impressions, faisaient connaître à leurs contemporains leur sentiment sur ce qu'ils venaient de visiter.

Arrêtons-nous à un voyage qui semble avoir eu un statut particulier tant il paraît avoir marqué les socialistes - et non des moindres - qui l'effectuèrent. Il s'agit du voyage accompli à l'occasion de l'inauguration de la nouvelle maison du peuple de Bruxelles, en avril $1899^{(16)}$. Ce voyage fut notamment effectué par les délégués de la Fédération des chambres syndicales ouvrières de la Seine-Inférieure ${ }^{(17)}$. Ce qui frappe tout d'abord, à la lecture du rapport de Hardy, du syndicat des lithographes, et Briot, du syndicat des cordonniers, c'est la forte impression que leur laissèrent la maison du peuple, la coopération socialiste belge et, plus globalement, le Parti ouvrier belge. Mais ce rapport est surtout significatif de ce type de démarche et d'écrit. En effet, Hardy et Briot souhaitaient qu'à la lecture de leur compte rendu les travailleurs de Seine-Inférieure et, au-delà, ceux du reste de la France partagent l'admiration qu'eux-mêmes avaient éprouvée devant les réalisations du socialisme belge. Plus encore, ils espéraient que le mouvement socialiste français trouverait une source d'inspiration dans ce mouvement ouvrier belge qui les avait tant impressionnés.

Si ce voyage en Belgique fit forte impression sur nos deux ouvriers de la Seine-Inférieure, le même voyage, la même inauguration furent loin de laisser indifférents les nombreux socialistes français qui, en réponse à l'invitation de leurs camarades belges, se rendirent à Bruxelles pour les fêtes d'avril 1899. Ainsi étaient notamment présents: Dejeante, député de Paris; Colly, délégué par le groupe socialiste du conseil municipal de Paris; Marie Bonnevial, déléguée par les syndicats féminins de Paris ; Lavigne, délégué du Parti ouvrier français; Bock, de L'Abeille suresnoise, Cublier, du Progrès de Gentilly, Guillemin, de L’Avenir de Plaisance, secrétaire de la Bourse coopérative des sociétés ouvrières, Marie, de L'Economie parisienne, Seigné, administrateur de L'Egalitaire, tous délégués par la Bourse 
(18) Le numéro du Peuple du lundi 3 avril 1899 note que la délégation parisienne aux fêtes inaugurales comptait cent membres.

(19) II est à noter que la Bourse a publié le compte rendu du voyage de la délégation dans son bulletin mensuel et que ce rapport sur la coopération belge fut tiré à 10000 exemplaires et diffusé à profusion malgré les problèmes financiers de la Bourse. Cf. J. Gaumont, Histoire générale de la coopération en France, op. cit., t. II, p. 293. (20) Cf. C. Prochasson, "Jean Jaurès et la coopération ", Revue de l'économie sociale, $n^{\text {os }} 3$ et $4,1985$.

(21) Discours du 2 avril 1899, Rapport des délégués de la Fédération des chambres syndicales ouvrières de la Seine-Inférieure, op. cit., p. 12. $C^{\prime}$ est nous qui soulignons.

(22) J. Jaurès, "La leçon des fêtes de Bruxelles ", Le Mouvement socialiste, $n^{\circ} 8,1899$, p. 450.

(23) N. Pilhes, Socialisme et coopération, de la révolution au réformisme (1879-1914), mémoire de maîtrise d'histoire, sous la direction de Maurice Agulhon, Paris-I, 1983, p. 236. coopérative des sociétés ouvrières ${ }^{(18)}$. Ces derniers furent tellement impressionnés par le spectacle de la nouvelle maison du peuple que, sous leur impulsion, les militants de la Bourse prirent dès lors pour modèle les méthodes de la coopération socialiste belge et s'efforcèrent de les faire adopter par les coopératives françaises ${ }^{(19)}$.

Ayant pris également part aux fêtes inaugurales de la nouvelle maison du peuple, Jaurès revint conquis par le modèle belge de la coopération ${ }^{(20)}$. Dans les deux discours qu'il prononça lors des cérémonies bruxelloises, non seulement il rendit hommage aux œuvres du mouvement ouvrier belge et au caractère fondamentalement socialiste de la coopération d'outre-Quiévrain, mais encore il présenta le socialisme belge comme un exemple à suivre. Il s'engagea à offrir à l'imitation de ses camarades, dès son retour en France, le modèle admiré en Belgique: "Eh bien, nous vous promettons d'aller prê-cher votre exemple à nos frères, les initier à l'organisation, à la coopération, et de mettre la classe ouvrière en état de gouverner et d'administrer le monde (21) !" Dans le prolongement de ses discours bruxellois, Jaurès tira la "leçon des fêtes de Bruxelles » dans un article qu'il écrivit pour le numéro du $1^{\text {er }}$ mai 1899 du Mouvement socialiste. Fort des enseignements tirés de l'exemple belge ${ }^{(22)}$, il revenait sur la nécessité de développer un mouvement coopératif tout pénétré de socialisme et invitait les socialistes français à créer partout des coopératives. Ainsi le voyage en Belgique apparait-il comme une étape essentielle sur la voie qui a conduit Jaurès à reconnaître la valeur de la coopération. L'exemple belge a largement contribué à le convaincre que la coopération était non seulement efficace, mais aussi nécessaire à l'émancipation du prolétariat.

S'il fut important, voire déterminant, le voyage d'avril 1899 à Bruxelles ne fut pas le seul voyage d'études effectué par des socialistes français en Belgique. Si les socialistes coopérateurs ont voulu connaître la coopération anglaise, s'ils ont cherché à visiter l'organisation de la Wholesale de Manchester, les affinités plus grandes des mouvements coopératifs socialistes belge et français entraînaient encore la plupart des voyageurs vers la coopération de Wallonie et des Flandres. Ainsi, parti en 1908 visiter les socialistes d'outre-Quiévrain, Albert Thomas eut une expression significative: il disait être allé "prendre une leçon de coopération socialiste ${ }^{(23)}$ ". S' exprimant ainsi, il résumait la pensée de nombre des socialistes qui firent en ce début de siècle le voyage de Belgique.

Le voyage en Belgique a donc non seulement représenté l'une des modalités de contact direct entre socialistes français et belges, mais aussi constitué un canal par lequel se sont développées des influences. En fait, ce vecteur d'influences présente une double face: les influences s'exerçaient d'abord sur place sur le voyageur; puis, dans un deuxième temps, elles pouvaient agir plus largement par l'intermédiaire d'écrits. C'est ainsi que la pensée de certains socialistes français en matière de coopération a pu s'enrichir et évoluer au contact des réalités observées en Belgique même. De cette rencontre avec le mouvement ouvrier belge, avec ses dirigeants, ses militants et ses réalisations, les voyageurs rapportaient des images, des impressions, des 
(24) Le premier numéro du Mouvement socialiste parut le 15 janvier 1899 et la revue disparut à la veille du premier conflit mondia (juin 1914). Hubert Lagardelle en était le directeur en 1899 et il assura, seul et sans que son autorité fût jamais remise en question, la direction de la revue jusqu'à sa disparition.

(25) De 1899 - date de sa première collaboration - à 1912, nous en avons recensé vingt-quatre. enseignements quils ont pu répandre parmi leurs camarades en donnant un prolongement écrit à leur voyage (publication de récits, rapports et autres comptes rendus). Et il nous semble que les relations des voyageurs français en terre belge ont joué un rôle dans la transformation de l'idée que les socialistes français se faisaient de la coopération et dans la conversion de certains au mouvement coopératif.

\section{Des revues comme lieux d'échanges}

Enfin deux revues, l'une française - Le Mouvement socialiste -, l'autre belge - l'Avenir social-, qui apparaissent comme des lieux de rencontres et d'échanges pour les socialistes des deux pays nous ont semblé pouvoir constituer également des vecteurs d'influences. Parmi les revues socialistes qui fleurirent durant la dernière décennie du XIX siècle, Le Mouvement socialiste ${ }^{(24)}$ bénéficia d'un prestige - lié notamment à la qualité de sa documentation et de ses collaborateurs français et étrangers - et d'une audience certains en France comme à l'étranger. Revue mensuelle dont le Parti ouvrier belge s'est doté en mai 1896 et qui cessa de paraitre en février 1907, société coopérative affiliée au POB, l'Avenir social s'était voulu la revue doctrinale du socialisme belge, ce qui ne l'empêcha pas d'être en même temps le bulletin de la Fédération des conseillers communaux, de la Fédération des sociétés coopératives et de la commission syndicale du POB. Mais si les deux revues contribuèrent à faire connaitre le mouvement coopératif voisin, si elles peuvent apparaitre comme des lieux d'interaction entre socialistes et coopérateurs belges et français, l'intensité des échanges et le poids qu'ils purent avoir ne paraissent pas avoir été identiques. S'il y eut un vecteur des influences du socialisme belge sur celui de France en matière coopérative, il nous semble qu'il fut plus nettement constitué par la revue de Lagardelle, dont les collaborateurs formaient un authentique milieu.

Deux constantes apparaissent tout au long de l'existence du Mouvement socialiste: son ouverture sur le monde et une orientation coopérative qui ne se démentit pas jusqu'en 1914. Parmi les pôles étrangers en direction desquels Le Mouvement développa sa collaboration, la Belgique se dégage tout particulièrement. En effet, à côté du comité de rédaction pour la France et de l'adresse parisienne de la revue figuraient un comité de rédaction pour la Belgique, composé d'Emile Vandervelde, de Max Hallet et de Gaston Vandermeeren, et une adresse bruxelloise. Même si le comité de rédaction propre à la Belgique ne semble pas avoir brillé par son activité, même si son existence fut brève (il disparut en 1902), cela n'ôte rien aux relations particulières qu'entretinrent socialistes belges et français au sein du Mouvement socialiste. Emile Vandervelde devait devenir un collaborateur important de la revue; important en raison de son prestige, mais aussi par la quantité d'articles qu'il signa ${ }^{(25)}$. Même si ce ne fut pas dans des proportions semblables, plusieurs camarades de Vandervelde l'imitèrent (Edouard Anseele, Louis Bertrand, Louis De Brouckère, Jules Destrée, Auguste Dewinne et Gaston Vandermeeren, tous dirigeants du Parti ouvrier belge), écrivant plus ou moins régulièrement dans la revue de Lagardelle. 
(26) J. Jaurès, " La leçon des fêtes de Bruxelles ", loc. cit.

(27) A. Dewinne, "La coopération en Belgique ", $\mathrm{n}^{\text {os }} 16$ et 17,1899 ; E. Vandervelde, "La nouvelle maison du peuple de Bruxelles ", $n^{\circ} 6$, 1899 ; E. Vandervelde, "Syndicats agricoles et coopératives socialistes " $n^{\circ} 55,1901$.

(28) E. Anseele, "La coopération socialiste ", n 126, 1903; "La coopération et le socialisme ", $\mathrm{n}^{\circ \mathrm{s}} 39,1900$, et $217,1910$.
L'autre constante fut l'orientation coopérative du Mouvement. Attaché à promouvoir l'action économique du mouvement ouvrier, reconnaissant une formule d'essence socialiste dans la coopération, Le Mouvement socialiste s'efforça de faire partager ce point de vue, se livrant à un véritable travail d'éducation. L'ouverture sur les expériences étrangères, la référence aux analyses produites hors des frontières nationales, bref, le souci international de la revue de Lagardelle se retrouve en matière coopérative. Là encore la prééminence belge se constate. Attirés et séduits par les méthodes de la coopération socialiste belge, les hommes du Mouvement socialiste ont avant tout dirigé leurs investigations et leurs enquêtes vers elle. C'est elle qu'ils ont d'abord fait connaître à leurs lecteurs par leurs articles; ils se sont employés à montrer les résultats obtenus par les grandes coopératives ouvrières de Gand, de Bruxelles, de Jolimont, sur lesquelles ont été publiés chiffres et monographies. Nous avons vu que ce fut dans leur revue que Jaurès, rentrant de Bruxelles impressionné par le spectacle offert par la coopération belge, avait appelé les socialistes à créer partout des coopératives animées de l'esprit socialiste ${ }^{(26)}$. Dans Le Mouvement, le socialiste belge Auguste Dewinne a décrit la coopération de son pays; Emile Vandervelde a présenté, à la veille de son inauguration, la nouvelle maison du peuple de Bruxelles et a consacré un article aux relations entre syndicats agricoles et coopératives socialistes ${ }^{(27)}$. Lagardelle a fait appel à la réflexion - nourrie par la pratique - des socialistes d'outre-Quiévrain: Edouard Anseele a ainsi étudié "La coopération socialiste » et les rapports de la coopération et du socialisme ${ }^{(28)}$; et sur les neuf socialistes français et étrangers à qui il fut demandé de réfléchir sur "La coopération et le socialisme ", quatre étaient belges (Edouard Anseele, Louis Bertrand, Camille Huysmans et Alphonse Octors). Mu par un souci de documentation et d'éducation, attaché au développement de la coopération, Le Mouvement socialiste a favorisé la rencontre des socialistes belges et français, le contact des expériences et des réflexions des uns et des autres. Ce faisant, il nous semble avoir joué un rôle non négligeable dans l'orientation de nombre de socialistes dans la voie coopérative.

\section{La coopération: un modèle offert par le socialisme belge}

\section{La coopération, base du mouvement ouvrier belge}

A la fin du XIX siècle et jusqu'en 1914, le Parti ouvrier belge a représenté un modèle au sein du mouvement ouvrier européen. Si la plupart des partis sociaux-démocrates en formation ont emprunté au SPD allemand son programme, ils se sont davantage inspirés du modèle belge. Incarné par le $\mathrm{POB}$, ce modèle était tout d'abord caractérisé par la conjonction des fonctions d'encadrement, de service et d'expression du mouvement ouvrier. Le Parti ouvrier se présentait sous la forme d'une organisation " englobante »; en son sein se concentraient les divers aspects du mouvement ouvrier: l'action politique représentée par les ligues ouvrières, le syndicalisme, le 
(29) Des coopératives apparurent à Bruxelles (La Boulangerie coopérative ouvrière, fondée en 1881 , qui devint la maison du peuple en 1886), à Jolimont (Le Progrès) et à Louvain (Les Prolétaires) en 1886, à Liège (La Populaire) en 1887, à Verviers (La Ruche ouvrière) en 1888, à Roux (La Concorde) en 1891 à Waremme (La Justice) en 1898, etc. mouvement mutualiste, les associations culturelles et surtout la coopération. Et si la liaison organique établie entre ces différentes branches du mouvement ouvrier faisait la réputation du socialisme belge, son originalité lui venait particulièrement de la place que tenait le mouvement coopératif dans l'organisation socialiste, de l'extraordinaire développement de la boulangerie coopérative et de la maison du peuple. De la création du POB à la Première Guerre mondiale, la coopération constituait la véritable épine dorsale de l'organisation socialiste.

Si le mouvement coopératif a connu une véritable explosion en Belgique entre 1885 et 1914, la véritable naissance de la coopération socialiste est antérieure à la création du Parti ouvrier, puisqu' elle correspond à la fondation du Vooruit en 1880 par les coopérateurs socialistes emmenés par Edouard Anseele et Edmond Van Beveren. Première coopérative entièrement socialiste, le Vooruit montra le chemin et servit de modèle aux autres coopératives ouvrières belges. Suite au développement et aux succès du Vooruit, des dizaines de coopératives de tendance socialiste virent le jour dans les centres industriels du pays ${ }^{(29)}$. A la multiplication de ces coopératives socialistes répondit celle des maisons du peuple: si la première était apparue en 1872 à Jolimont, on en comptait 66 en 1893 et 149 en 1914. Chaque coopérative, dès que ses moyens le lui permettaient, voulait avoir la sienne, si bien que chaque village important, ou presque, eut sa maison du peuple. Toute la vie du mouvement socialiste s'y concentrait : la maison du peuple abritait bien sûr la coopérative, mais aussi la mutuelle, le syndicat et le parti.

\section{Le poids de l'exemple belge chez les socialistes français}

Or, au début de la période qui nous intéresse, le mouvement ouvrier français apparaît comme très réticent face à l'idée de coopération. Pour les socialistes, l'essentiel résidait dans l'action politique. Toutefois, leur position à l'égard de la coopération évolua au cours de la dernière décennie du siècle. S'ils s'investirent alors bien davantage dans le mouvement coopératif, si l'opinion qu'ils s'en faisaient se modifia, c'est en raison même de l'influence qu'ont pu avoir sur eux les exemples de réussite de coopératives socialistes; et s'ils furent marqués, ce fut, en premier lieu, par l'expérience belge. Nous l'avons vu, les socialistes français qui avaient assisté aux fêtes inaugurales de la nouvelle maison du peuple de Bruxelles, en avril 1899, étaient revenus de Belgique fortement impressionnés par ce qu’ils y avaient vu. Les uns et les autres, séduits, conquis par le modèle belge de la coopération l'offrirent en exemple à leurs camarades.

Ainsi, à la suite du voyage en Belgique d'avril 1899, la coopération belge devint le modèle auquel les socialistes français n'allaient plus cesser de se référer. Les militants de la Bourse coopérative des sociétés ouvrières de consommation instituèrent en modèle les méthodes de la coopération socialiste belge et s'efforcèrent de les faire adopter par les coopératives françaises. D'ailleurs, dans la " Notice sur la Bourse coopérative " annexée au compte rendu du I ${ }^{\mathrm{er}}$ Congrès national et international de la coopération socialiste, 
(30) X. Guillemin, « Notice sur la Bourse coopérative ", Premier Congrès national et international de la coopération socialiste tenu à Paris les 7, 8, 9 et 10 juillet 1900, compte rendu, Société nouvelle de librairie et d'édition (Librairie Georges Bellais), Paris, 1900, p. 206.

(31) Cité par P. Brizon, E. Poisson, "La coopération ", Encyclopédie socialiste, op. cit., p. 217.

(32) Cité par P. Brizon, E. Poisson, "La coopération ", Encyclopédie socialiste, op. cit., p. 217. son secrétaire, Xavier Guillemin, notait pour l'année 1899: "Une des plus importantes initiatives de la Bourse en cette année a été l'organisation de la délégation à l'inauguration de la maison du peuple de Bruxelles. Le rapport publié à cet effet dans le numéro 24 du Bulletin a été répandu par toute la France et a servi à lancer les coopératives dans la voie socialiste ${ }^{(30)}$."

Les militants de la Bourse ne tardèrent pas à mettre en application ce qu'ils avaient appris de l'exemple belge. Etudiant la création de boulangeries coopératives dans les divers quartiers de Paris, Xavier Guillemin chargea une commission de réaliser, dans le XIII ${ }^{\mathrm{e}}$ arrondissement, un projet de maison du peuple sur le modèle bruxellois et sur celui du Vooruit de Gand; une maison du peuple qui, comme toutes les sociétés belges ou presque, serait basée sur une boulangerie coopérative. S'inspirant des conceptions et méthodes appliquées en Belgique, la Boulangerie socialiste, installée rue Barrault en 1899, devait être d'après ses fondateurs - parmi lesquels figuraient Xavier Guillemin, Alfred Hamelin, Philippe Landrieu et Marcel Mauss - un exemple de construction pratique de coopération socialiste. Toutefois, c'était dans ce département du Nord où avaient immigré tant de travailleurs belges qu'était apparue, quelques années plutôt, la première coopérative socialiste de France. Fondée à Roubaix en 1885 par des ouvriers belges travaillant en France, selon le modèle en application à Gand, La Paix a été considérée comme le "Vooruit de la France du Nord ${ }^{(31)}$ ". Si, comme en Belgique et en Allemagne, la grande coopérative gantoise a constitué en France un modèle, cet exemple a d'abord été suivi dans le nord du pays. Or, ceci se produisit dans une région dont la principale organisation socialiste, le Parti ouvrier français (POF) de Jules Guesde, apparaissait, en cette fin de XIX ${ }^{e}$ siècle, comme la tendance du socialisme français qui marquait le plus son hostilité à la coopération. Mais s'il était un adversaire théorique de la coopération, Guesde ne reconnaissait pas moins, dès 1884, qu'entre de bonnes mains les coopératives pouvaient avoir quelque résultat intéressant. Ces «bonnes mains ", il les apercevait d'abord de l'autre côté de la frontière: "Ce qui ne veut pas dire qu'exceptionnellement, dans certaines conditions et entre certaines mains, les coopératives de consommation ne puissent rendre des services aux travailleurs. Il n'y a, pour se convaincre du contraire, qu’à voir le parti qu'a su en tirer le socialisme flamand. " Reconnaissant le rôle positif joué par les dirigeants du Vooruit, il ajoutait que «le pain à meilleur marché n'a été qu'un moyen de grouper les hommes et de véhiculer les idées ${ }^{(32)}$ ". Si les socialistes du Nord, les guesdistes ont été, plus encore que leurs camarades, hostiles à la coopération, leur attitude n'en a pas moins évolué et ce changement a tenu, plus encore qu'ailleurs sans doute, à la force de l'exemple belge.

Un autre exemple de coopérative créée sur le modèle belge nous est fourni par L'Union de Lille. D'ailleurs, pour la présenter, les deux auteurs du volume "Coopération " de l'Encyclopédie socialiste recourent une nouvelle fois à la référence belge: "Comme le Vooruit a été dépassé par la maison du peuple de Bruxelles, fondée après lui, La Paix a été dépassée par L'Union de Lille ouverte en $1892^{(33)}$. " Puis, racontant la création de la coopérative, 
(34) Ibid., p. 219

(35) Ainsi furent créées L'Avenir des ouvriers d'Armentières, La Fraternelle d'Halluin, L'Union d'Houplines, La Solidarité ouvrière de Tourcoing, L'Humanité de Wattrelos, etc.

(36) Nom qu'avait pris la Bourse à la suite de son congrès de Calais (1911).

(37) E. Vandervelde, La coopération neutre et la coopération socialiste, Librairie Félix Alcan, Paris, 1913, p. 95.

(38) Citons le cas de Georges Sorel qui voyait dans la coopération un idéal vicié par ses origines bourgeoises.

(39) H. De Man, "La particularité du mouvement ouvrier en Belgique ", Le mouvement ouvrier en Belgique, Editions de la Fondation Joseph Jacquemotte, Bruxelles, 1965, p. 65. De Man ajoutait: « Nous appellerons crétinisme coopératif cette mentalité et cette conception particulière engendrées par le développement hypertrophique du coopératisme. " ils notent - fait significatif - qu' "elle prit naissance au milieu d'un groupe [d'une dizaine d'amis], parmi lesquels se trouvaient quelques camarades revenus tout récemment d'un voyage quills venaient d'effectuer en Belgique et où ils avaient admiré l'organisation des grandes coopératives ouvrières de Gand et de Bruxelles ${ }^{(34)}$ ". Comme on peut le constater, le voyage en Belgique eut, plus d'une fois, une influence déterminante. A l'image des coopératives belges les plus importantes, L'Union diversifia ses activités tout en se développant et, surtout, devint le cœur de l'organisation ouvrière lilloise. Quant aux cités industrielles du département du Nord, elles ont toutes ou presque constitué leur coopérative ${ }^{(35)}$, à l'exemple de La Paix et de L'Union et, plus ou moins directement, sur le modèle du Vooruit.

Ainsi, du début à la fin de la période qui nous intéresse, l'opinion des socialistes français à l'égard de la coopération évolua sensiblement sous l'influence du modèle qu' offraient la coopération belge et ses deux plus beaux fleurons: le Vooruit de Gand et la maison du peuple de Bruxelles. En prenant connaissance des résultats obtenus par les sociétés belges, nombre d'entre eux en vinrent à reconnaitre l'utilité de l'outil coopératif pour l'action socialiste.

Il y eut donc bien influence du socialisme belge sur le socialisme français dans le domaine coopératif. Empruntant différents canaux, porté par divers vecteurs, le modèle de la coopération belge attira l'attention de socialistes français qui, bien souvent, s'en inspirèrent. Néanmoins, il est un point au moins sur lequel Emile Vandervelde ne reconnut pas l'influence du socialisme belge: ce fut cette unité qui se réalisa en décembre 1912 entre la Confédération coopérative ${ }^{(3)}$ et l'Union; unité qui, sous la forme d'un compromis plutôt favorable aux neutralistes, donna naissance à la Fédération nationale des coopératives de consommation et fit dire au dirigeant du POB qu' " il y a donc, incontestablement, en France, une tendance à réaliser l'unité coopérative, mais c'est la coopération socialiste qui fait, à peu près, tous les frais du rapprochement des deux écoles ${ }^{(37)}$ ». Par ailleurs, et malgré sa valeur et sa force, l'exemple belge n'éteignit pas les réticences, la méfiance que certains socialistes français éprouvaient à l'égard de la coopération ${ }^{(38)}$. Mais de telles critiques n'étaient pas réservées à la France et, quand Sorel qualifiait le socialisme belge de "socialisme où l'épicerie [est] érigée en sacerdoce ", Henri De Man n’hésitait pas à parler de "crétinisme coopératif ${ }^{(39)}$ ". Ainsi, au sein même du Parti ouvrier belge, des socialistes soulignèrent les néfastes effets de la coopération. Il est vrai que le rôle primordial qu'ont joué les coopératives dans la constitution du POB a souvent été considéré comme un signe particulièrement révélateur du pragmatisme et de l'orientation réformiste qu'adopta ce parti dès sa naissance. Avec le développement du mouvement coopératif se mit en place une bureaucratie ouvrière qui, soucieuse de la gestion des sociétés, était peu encline à risquer le sort de son organisation dans l'aventure révolutionnaire. A l'image du mouvement socialiste belge, la coopération présentait un visage plus pragmatique que doctrinal. Et, en raison même de son poids au sein de l'organisation socialiste, elle contribua largement à renforcer l'orientation réformiste et le pragmatisme de ce mouvement. 